\title{
A Numerical Solutions Based on the Quasi-wavelet Analysis*
}

\author{
Z.H. Huang ${ }^{1}$, L. Xia ${ }^{2}$, and X.P. $\mathrm{He}^{3}$ \\ ${ }^{1}$ College of Computer Science, Chongqing Technology and Business University, Chongqing, \\ P.R. China \\ zhhuangctbu@yahoo.com.cn \\ ${ }^{2}$ College of Science, Chongqing Technology and Business University, Chongqing, 400067, \\ P.R. China \\ xl@etbu.edu.cn \\ ${ }^{3}$ College of Computer Science, Chongqing Technology and Business University, Chongqing, \\ 400067, P.R. China \\ jsjhxp@ctbu.edu.cn
}

\begin{abstract}
Taking the approximate equations for long waves in shallow water as example, the quasi-wavelet discrete scheme is proposed for obtaining numerical solution of the $(1+1)$ dimension nonlinear partial differential equation. In the method, the quasi-wavelet discrete scheme is adopted to discretize the spatial derivative discrete and the ordinary differential equation about time is obtained. Then the fourth order Rung-Katta method is employed to discretize the temporal derivative. Finally the quasi-wavelet solution is compared with the analytical solution, and the computations are validated.
\end{abstract}

Keywords: interval quasi_shannon; precise integration method; approximate equations of long waves in shallow water.

\section{Introduction}

In recent years, many methods have been developed for the analytical solution to nonlinear partial differential equation (PDE). For example, important such ones as homogeneous balance method, variable separable methods and Jacobi elliptic function expansion are used to solve PDE exact solution and solitary wave solution[1-5]. But in general, it is quite difficult to carry on a deep study to the wellposedness of the solutions. Wavelet function is the energy function with locality characterization, and it is characterized by utilizing information from the nearest neighboring grid pints to approximate the differentiation at a point, and thus is much more flexible. The wavelet method is applied to numerical solution of PDE by Morlet J, Arens G, Fourgeau E, et al. Its applications have become one of the fastest growing research areas. The

\footnotetext{
* This work was supported by the Key Science-Technology Project of Chongqing under Grant NO.CSTC-2005AC2090, and the Science Foundation of Chongqing under Grant NO.CSTC$2006 \mathrm{BB} 2249$.
} 
Daubechies scaling function constructs the grid point method of wavelet to solve PDE by [6]. Daubechies scaling function converges slowly with respect to mesh refinement and is not cost-effective for achieving high precision. Hence, there is a strong demand for a scheme, which can solve the numerical solutions of nonlinear partial differential equations. The scaling function of Shannon's wavelet is represented in an analytical form. When Shannon scaling is multiplied by Gauss function[7], a function, quasi scaling function will be obtained. The wavelet of orthogonal function when it is further generalized is called quasi-wavelet. While high-accuracy solution of numerical is very useful to nonlinear PDEs when it functions as a local method.

The numerical solution of PDE is solved by method of quasi-wavelet in [8]. However, research of application of quasi-wavelet to numerical solutions of nonlinear PDEs has not been conducted, for example $(1+1)$ dimensional models such as the approximate equations for long waves in shallow water have not been studied. This paper uses the quasi-wavelet method to construct scaling function. In the method the quasi-wavelet discrete scheme is adopted to discretize the spatial derivatives, and the fourth order Rung-Katta method is adopted to discretize the temporal derivatives. The numerical solution of example showed that quasi-wavelet method is a success in computation of the nonlinear PDEs solutions, and the method is helpful to improve the precision of the solutions. In the end, the computation results are validated based on the numerical solutions of the quasi-wavelet and the analytical solutions.

\section{Quasi-wavelet Solutions of Approximate Equations for Waves in Shallow Water}

The long waves equations in shallow water have been found by Whithom and Broer

$$
\left\{\begin{array}{l}
u_{t}-u u_{x}-v_{x}+\frac{1}{2} u_{x x}=0 \\
v_{t}-(u v)_{x}-\frac{1}{2} v_{x x}=0
\end{array}\right.
$$

initial-boundary condition

$$
\begin{array}{lr}
u(a, t)=u_{1}(t), & u(b, t)=u_{2}(t) \\
v(a, t)=v_{1}(t), & v(b, t)=v_{2}(t)
\end{array}
$$

Where $x \in[a, b] \quad t>0$

\subsection{Spatial Coordinate Discrete to the Long Waves in Shallow Water}

Identically proportion of an $\mathrm{x}$-coordinate of spatial, $\Delta$ is the grid spacing. $\Delta x=(b-a) / N$, where $\mathrm{N}$ is the total computational narrow bandwidth [a, b]. The $\left\{x_{i}=a+(i-1) \Delta x\right\} \quad(\mathrm{i}=1,2,3 \ldots \ldots \mathrm{N}+1)$ refers to discrete sampling pints centered 
around the point $\mathrm{x}$, and $x_{i}-x_{i+k}=k \Delta x$, the function values $\left\{u_{i}\right\}$ and $\left\{v_{i}\right\}$ with respect to the coordinate at grid point $x_{i}$, the Eq.(1)-(2) are represented as follows

$$
\begin{aligned}
& \frac{\partial u_{i}}{\partial t}=-\frac{1}{2} \frac{\partial^{2} u_{i}}{\partial x^{2}}+u_{i} \frac{\partial u_{i}}{\partial x}+\frac{\partial v_{i}}{\partial x} \\
& \frac{\partial v_{i}}{\partial t}=\frac{1}{2} \frac{\partial^{2} v_{i}}{\partial x^{2}}+u_{i} \frac{\partial v_{i}}{\partial x}+v_{i} \frac{\partial u_{i}}{\partial x}
\end{aligned}
$$

Let

$$
\begin{aligned}
& f_{i}=-\frac{1}{2} \frac{\partial^{2} u_{i}}{\partial x^{2}}+u_{i} \frac{\partial u_{i}}{\partial x}+\frac{\partial v_{i}}{\partial x} \\
& g_{i}=\frac{1}{2} \frac{\partial^{2} v_{i}}{\partial x^{2}}+u_{i} \frac{\partial v_{i}}{\partial x}+v_{i} \frac{\partial u_{i}}{\partial x}
\end{aligned}
$$

$(\mathrm{i}=1,2,3, \ldots \ldots . . \mathrm{N}+1)$

By (5)-(8), Eq.(5)-(6)can be expressed as follows

$$
\left\{\begin{array}{l}
\frac{d u_{i}}{d t}=f_{i} \\
\frac{d v_{i}}{d t}=g_{i}
\end{array}\right.
$$

\subsection{Numerical Discrete Forms of Quasi-wavelet Spatial Coordinate Discrete to the Long Waves in Shallow Water}

To solve Eq.(9)-(10), the regularized Shannon's delta kernel is used, which can dramatically increase the regularity of Shannon's wavelet scaling function,[or quasiscaling function][8].

$$
\delta_{\Delta, \sigma}(x)=\frac{\sin (\pi x / \Delta)}{\pi x / \Delta} \exp \left[-x^{2} / 2 \sigma^{2}\right]
$$

where $\Delta$ is the grid spacing, $\sigma$ is determines the width of the Gauss envelop and can be varied in association with the grid space. i.e., $\sigma=r \Delta$ and $r \geq \sqrt{2} / \pi, \mathrm{r}$ is the parameter. The regularized Shannon's wavelet is called quasi-wavelet. Shannon's scaling function is recognized as basis function, the function $f(x)$ and $g(x)$ in the interval $\left[-\frac{\pi}{\Delta}, \frac{\pi}{\Delta}\right]$ can be expressed as

$$
\begin{aligned}
& f(x)=\sum_{k=-\infty}^{+\infty} \delta_{\Delta, \sigma}\left(x-x_{k}\right) f\left(x_{k}\right) \\
& g(x)=\sum_{k=-\infty}^{+\infty} \delta_{\Delta, \sigma}\left(x-x_{k}\right) g\left(x_{k}\right)
\end{aligned}
$$


In discrete singular convolution algorithm, the band-limits $f(x), g(x)$ and its derivatives with respect to the coordinate at grid point $\mathrm{x}$ are approximated by linear sun of discrete values $\left\{f\left(x_{k}\right)\right\}$ and $\left\{g\left(x_{k}\right)\right\}$ in the interval $\left[-\frac{\pi}{\Delta}, \frac{\pi}{\Delta}\right]$

$$
\begin{aligned}
f^{(n)}(x) & \approx \sum_{k=-w}^{w} \delta_{\Delta, \sigma}^{(n)}\left(x-x_{k}\right) f\left(x_{k}\right),(n=0,1,2 \ldots \ldots) \\
g^{(n)}(x) & \approx \sum_{l=-w}^{+w} \delta_{\Delta, \sigma}^{(n)}\left(x-x_{k}\right) g\left(x_{k}\right),(n=0,1,2 \ldots \ldots \ldots)
\end{aligned}
$$

In fact, the $2 \mathrm{w}$ point of computation is obtained in the coordinate around a grid point $\left\{x_{i}\right\} ; 2 \mathrm{w}+1$ is the total computational bandwidth, which is usually much smaller than the computational domain. Eq.(15)-(16) are called quasi-wavelet form of numerical discrete.

To compute Eq.(14)-(15), the regularized Shannon's delta kernel is used, the delta expressions for (14)-(15) $\delta_{\Delta}^{(1)}$ and $\delta_{\Delta}^{(2)}$ can be given analytically as

$$
\begin{aligned}
& \delta_{\Delta, \sigma}^{(1)}(\mathrm{x})=\left\{\begin{array}{lc}
\mu\left(\frac{\kappa}{x}-\frac{\lambda \Delta}{\pi x^{2}}-\frac{\lambda \Delta}{\pi \sigma^{2}}\right) & x \neq 0 \\
0 & x=0
\end{array}\right. \\
& \delta_{\Delta, \sigma}^{(2)}(x)= \begin{cases}\mu\left[\lambda\left(-\frac{\pi}{\Delta x}+\frac{2 \Delta}{\pi x^{3}}+\frac{\Delta}{\pi x \sigma^{2}}+\frac{\Delta x}{\pi \sigma^{4}}\right)-2 \kappa\left(\frac{1}{x^{2}}+\frac{1}{\sigma^{2}}\right)\right] & , x \neq 0 \\
-\frac{3+\pi^{2} \sigma^{2} / \Delta^{2}}{3 \sigma^{2}} & , x=0\end{cases}
\end{aligned}
$$

Where $\mu=\exp \left[-x^{2} / 2 \sigma^{2}\right], \lambda=\sin (\pi x / \Delta)$ and $\kappa=\cos (\pi x / \Delta)$.

\subsection{Temporal Derivatives Discretization}

A Rung-Kutta schema is used for temporal derivatives. The ordinary differential Eqs. (9)-(10) are used by fourth-order Rung-Katta method to discretize temporal derivatives, which can be expressed as follows

$$
u_{i}^{n+1}=u_{i}^{n}+\frac{\Delta t}{6}\left[K_{i, 1}+2 K_{i, 2}+2 K_{i, 3}+K_{i, 4}\right] \quad(i=1,2,3, \ldots \ldots . N=1)
$$

where

$$
\begin{aligned}
& K_{i, 1}=f_{i, 1}^{n} \quad K_{i, 2}=f_{i, 2}^{n} \quad K_{i, 3}=f_{i, 3}^{n} \quad K_{i, 4}=f_{i, 4}^{n} \quad(\mathrm{i}=1,2, \ldots, \mathrm{N}+1) \\
& v_{j}^{n=1}=v_{j}^{n}+\frac{\Delta t}{6}\left[L_{j, 1}+2 L_{j, 2}+2 L_{j, 3}+L_{j, 4}\right] \\
& L_{j, 1}=g_{j, 1}^{n} \quad L_{j, 2}=g_{j, 2}^{n} \quad L_{j, 3}=g_{j, 3}^{n} \quad L_{j, 4}=g_{j, 4}^{n} \quad(\mathrm{j}=1,2, \ldots, \mathrm{N}+1)
\end{aligned}
$$

Where upper sign $\mathrm{n}$ is time level, $\Delta t$ is length of time.

From Eq.(16), we have 


$$
\begin{aligned}
& K_{i, 1}=f_{i, 1}^{n}=-\frac{1}{2} \sum_{m=-w}^{w} \delta_{\Delta, \sigma}^{(2)}(-m \Delta x) u_{m+i}^{n}+u_{i}^{n} \sum_{m=-w}^{w} \delta_{\Delta, \sigma}^{(1)}(-m \Delta x) u_{i+m}^{n} \\
& +\sum_{m=-w}^{w} \delta_{\Delta, \sigma}^{(1)}(-m \Delta x) v_{i+m}^{n} \\
& K_{i, 2}=f_{i, 2}^{n}=-\frac{1}{2} \sum_{m=-w}^{w} \delta_{\Delta, \sigma}^{(2)}(-m \Delta x)\left[u_{m+i}^{n}+\frac{\Delta t}{2} K_{i+m, 1}\right]+\left[u_{i}^{n}+\frac{\Delta t}{2} K_{i, 1}\right] \\
& \times \sum_{m=-w}^{w} \delta_{\Delta, \sigma}^{(1)}(-m \Delta x)\left[u_{i+m}^{n}+\frac{\Delta t}{2} K_{i+m, 1}\right]+\sum_{m=-w}^{w} \delta_{\Delta, \sigma}^{(1)}(-m \Delta x)\left[v_{i+m}^{n}+\frac{\Delta t}{2} L_{i+m, 1}\right] \\
& K_{i, 3}=f_{i, 3}^{n}=-\frac{1}{2} \sum_{m=-w}^{w} \delta_{\Delta, \sigma}^{(2)}(-m \Delta x)\left[u_{m+i}^{n}+\frac{\Delta t}{2} K_{i+m, 2}\right]+\left[u_{i}^{n}+\frac{\Delta t}{2} K_{i, 2}\right] \\
& \times \sum_{m=-w}^{w} \delta_{\Delta, \sigma}^{(1)}(-m \Delta x)\left[u_{i+m}^{n}+\frac{\Delta t}{2} K_{i+m, 2}\right]+\sum_{m=-w}^{w} \delta_{\Delta, \sigma}^{(1)}(-m \Delta x)\left[v_{i+m}^{n}+\frac{\Delta t}{2} L_{i+m, 2}\right] \\
& K_{i, 4}=f_{i, 4}^{n}=-\frac{1}{2} \sum_{m=-w}^{w} \delta_{\Delta, \sigma}^{(2)}(-m \Delta x)\left[u_{m+i}^{n}+\Delta t u_{i+m, 2}\right]+\left[u_{i}^{n}+\Delta t K_{i, 3}\right] \\
& \times \sum_{m=-w}^{w} \delta_{\Delta, \sigma}^{(1)}(-m \Delta x)\left[u_{i+m}^{n}+\Delta t K_{i+m, 3}\right]+\sum_{m=-w}^{w} \delta_{\Delta, \sigma}^{(1)}(-m \Delta x)\left[v_{i+m}^{n}+\Delta t L_{i+m, 3}\right] \\
& L_{j, 1}=g_{j, 1}^{n}=\frac{1}{2} \sum_{m=-w}^{w} \delta_{\Delta, \sigma}^{(2)}(-m \Delta x) v_{m+j}^{n}+u_{j}^{n} \sum_{m=-w}^{w} \delta_{\Delta, \sigma}^{(1)}(-m \Delta x) v_{j+m}^{n}+v_{j}^{n} \sum_{m=-w}^{w} \delta_{\Delta, \sigma}^{(1)}(-m \Delta x) v_{j+m}^{n} \\
& L_{j, 2}=g_{j, 2}^{n}=\frac{1}{2} \sum_{m=-w}^{w} \delta_{\Delta, \sigma}^{(2)}(-m \Delta x)\left[v_{j+m}^{n}+\frac{\Delta t}{2} L_{j+m, 1}\right] \\
& +\left[u_{j}^{n}+\frac{\Delta t}{2} K_{j .1}\right]\left[\sum_{m=-w}^{w} \delta_{\Delta, \sigma}^{(1)}(-m \Delta x) \times\left[v_{j+m, 1}^{n}+\frac{\Delta t}{2} L_{j+m, 1}\right]\right. \\
& +\left[v_{j}^{n}+\frac{\Delta t}{2} L_{j, 1}\right] \sum_{m=-w}^{w} \delta_{\Delta . \sigma}^{(1)}(-m \Delta x)\left[u_{j+m}^{n}+\frac{\Delta t}{2} K_{j+m, 1}\right] \\
& L_{j, 2}=g_{j, 2}^{n}=\frac{1}{2} \sum_{m=-w}^{w} \delta_{\Delta, \sigma}^{(2)}(-m \Delta x)\left[v_{j+m}^{n}+\frac{\Delta t}{2} L_{j+m, 1}\right] \\
& +\left[u_{j}^{n}+\frac{\Delta t}{2} K_{j, 1}\right]\left[\sum_{m=-w}^{w} \delta_{\Delta, \sigma}^{(1)}(-m \Delta x) \times\left[v_{j+m, 1}^{n}+\frac{\Delta t}{2} L_{j+m, 1}\right]\right. \\
& +\left[v_{j}^{n}+\frac{\Delta t}{2} L_{j, 1}\right] \sum_{m=-w}^{w} \delta_{\Delta . \sigma}^{(1)}(-m \Delta x)\left[u_{j+m}^{n}+\frac{\Delta t}{2} K_{j+m, 1}\right] \\
& L_{j, 3}=g_{j, 3}^{n}=\frac{1}{2} \sum_{m=-w}^{w} \delta_{\Delta, \sigma}^{(2)}(-m \Delta x)\left[v_{j+m}^{n}+\frac{\Delta t}{2} L_{j+m, 2}\right]+\left[u_{j}^{n}+\frac{\Delta t}{2} K_{j .2}\right] \\
& \sum_{m=-w}^{w} \delta_{\Delta, \sigma}^{(1)}(-m \Delta x) \times\left[v_{j+m}^{n}+\frac{\Delta t}{2} L_{j+m, 2}\right]+\left[v_{j}^{n}+\frac{\Delta t}{2} L_{j, 2}\right] \sum_{m=-w}^{w} \delta_{\Delta . \omega}^{(1)}(-m \Delta x)\left[u_{j+m}^{n}+\frac{\Delta t}{2} K_{j+m, 2}\right] \\
& L_{j, 4}=g_{j, 4}^{n}=\frac{1}{2} \sum_{m=-w}^{w} \delta_{\Delta, \sigma}^{(2)}(-m \Delta x)\left[v_{j+m}^{n}+\Delta t L_{j+m, 3}\right] \\
& +\left[u_{j}^{n}+\Delta t K_{j .3}\right] \sum_{m=-w}^{w} \delta_{\Delta, \sigma}^{(1)}(-m \Delta x) \times\left[v_{j+m}^{n}+\Delta t L_{j+m, 3}\right] \\
& +\left[v_{j}^{n}+\Delta t L_{j, 3}\right] \sum_{m=-w}^{w} \delta_{\Delta . \sigma}^{(1)}(-m \Delta x)\left[u_{j+m}^{n}+\Delta t K_{j+m, 3}\right]
\end{aligned}
$$


When $\mathrm{t}=0$, the values of $\left\{u_{i}^{n}\right\}$ and $\left\{v_{i}^{n}\right\} \quad(\mathrm{n}=0)$ are obtained by Eq.(1)-(4). This can be rewritten as

$$
\begin{aligned}
& u_{i}^{0}=\bar{u}(x) \quad(\mathrm{i}=1,2,3 \ldots \mathrm{N}+1) \\
& v_{j}^{0}=\bar{v}(x) \quad(\mathrm{j}=1,2,3 \ldots \ldots . \mathrm{N}+1)
\end{aligned}
$$

Where $[-\mathrm{w},+\mathrm{w}]$ is the computation bandwidth. The w may be an arbitrary constant to reduce computation in narrower bandwidth.

\section{Overall Solutions Scheme}

In the above, we have computed $\delta_{\Delta, \sigma}^{(1)}$ and $\delta_{\Delta, \sigma}^{(2)}$ by only depending on the spacing $\Delta$, therefore, when the grid spacing is provided, the coefficients need to be computed only once and can be used during the whole computation. The main steps of computation can be expressed as following:

Step 1. using the known initial value both for $u_{i}^{0}(\mathrm{i}=1,2,3 \ldots \mathrm{N}+1)$ and $v_{j}^{0}$ $(\mathrm{j}=1,2,3 \ldots \ldots \mathrm{N}+1)$ or time level values of previous time $u_{i}^{n}$ and $v_{j}^{n} \quad(\mathrm{i}, \mathrm{j}=1,2,3 \ldots$ $\mathrm{N}+1)$, and outside the computational domain are required extension.

Step 2. From Eq.(20)-(26), transformations of the specified grid point values $f_{i, 1}^{n}, f_{i, 2}^{n}, f_{i, 3}^{n}, f_{i, 4}^{n}$ and $g_{j, 1}^{n}, g_{j, 2}^{n}, g_{j, 3}^{n}, g_{j, 4}^{4}$ are obtained.

Step 3. By subtracting $\operatorname{Eq}(16)-(18)$ from second-step, the values are computed $u_{i}^{n+1}$ and $v_{i}^{n+1} \quad(\mathrm{i}, \mathrm{j}=1,2,3 \ldots \ldots \mathrm{N}+1)$.

Step 4. Repeating the above process, from the first-step to the third-step with being computational value $u_{i}^{n+1}$ and $v_{i}^{n+1} \quad(\mathrm{i}, \mathrm{j}=1,2,3 \ldots \mathrm{N}+1)$ and the boundary condition. It satisfies the relation: $t=t+\Delta t$ and $n=n+1$, until required time level is achieved.

\section{Comparison Computations}

Both quasi-wavelet numerical solutions and the analytical solutions are computed, by Eq.(1)-(2). Assuming that Eq.(1)-(2) satisfy the initial-boundary condition below

$$
\begin{array}{cc}
u(x, 0)=\frac{c}{2}\left(1+\tanh \frac{c}{2} x\right) & v(x, 0)=\frac{c^{2}}{4} \sec h^{2} \frac{c}{2} x \\
u(a, t)=\frac{c}{2}\left[1+\tanh \frac{1}{2}\left(a c+\frac{c^{2}}{2} t\right)\right] & v(a, t)=\frac{c^{2}}{2} \sec h^{2} \frac{1}{2}\left[a c+\frac{c^{2}}{2} t\right] \\
u(b, t)=\frac{c}{2}\left[1+\tanh \frac{1}{2}\left(c b+\frac{c^{2}}{2} t\right)\right] & v(b, t)=\frac{c^{2}}{2}\left[1+\tanh \frac{1}{2}\left(c b+\frac{c^{2}}{2} t\right)\right]
\end{array}
$$

We have analytical solutions to Eq.(1)-(2) 


$$
u=\frac{c}{2}\left[1+\tanh \frac{1}{2}\left(c x+\frac{c^{2}}{2} t\right)\right], \quad v=\frac{c^{2}}{4} \sec h^{2} \frac{1}{2}\left[c x+\frac{c^{2}}{2} t\right]
$$

where $\mathrm{c}$ is an arbitrary constant.

To analyze and compare the computations, the initial values $u_{i}^{0}$ and $v_{j}^{0}$ of discrete are obtained from Eq.(29), where

$$
\begin{aligned}
& u_{i}^{0}=\frac{c}{2}\left\{1+\tanh \frac{c}{2}[a+(i-1) \Delta x]\right\}, \quad v_{j}^{0}=\frac{c^{2}}{4} \sec h^{2} \frac{c}{2}[a+(j-1) \Delta x] \\
& (\mathrm{i}, \mathrm{j}=1,2,3 \ldots . \mathrm{N}+1)
\end{aligned}
$$

We shall compute the values of previous time level from the above $u_{i}^{n}$ and $v_{i}^{n}$ (i, $\mathrm{j}=1,2,3 \ldots . \mathrm{N}+1)$. We choose $\mathrm{c}=0.5$, computational bandwidth $\mathrm{W}=10$, orthonormal band $\sigma=3.2 \Delta$, computation domain[a, b] $=[-100,100]$, the number of grid $\mathrm{N}=200$, allowable time step $\Delta t=0.002$. These values are computed by method of quasiwavelet, respectively, and such a plot is given in Figure 1-4.

From Eq.(1)-(2), these figures are excellent agreement between the analytical solutions and quasi-wavelet numerical solutions.

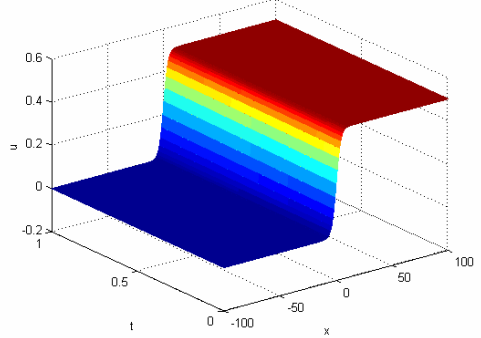

Fig. 1. u-analytical solutions

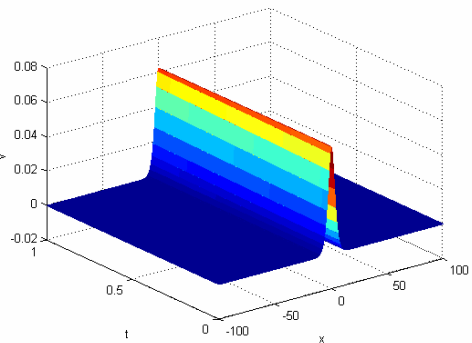

Fig. 3. v- analytical solutions

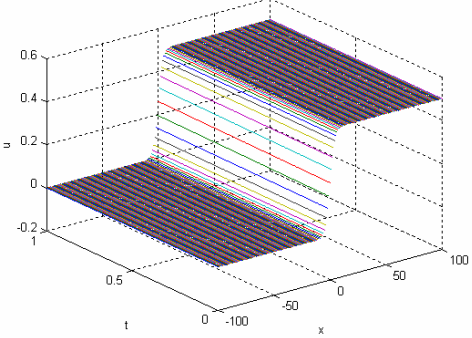

Fig. 2. u-quasi-wavelet solution (where $\mathrm{w}=10, \Delta \mathrm{t}=0.002, \sigma=3.2 \Delta$ )

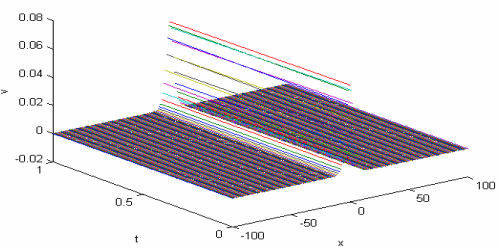

Fig. 4. v-quasi-wavelet solution (where $\mathrm{w}=10, \Delta \mathrm{t}=0.002, \sigma=3.2 \Delta$ ) 


\section{Conclusion}

In the paper, a new quasi-wavelets method for numerical application is introduced. In fact, their numerical solution is extremely approximate with analytical solutions and solving PDEs. The latter has won great success in wide application to various on PDEs.

\section{References}

1. Whutham, G. B.:Variational methods and applications to water waves. Proc Roy London, 1967:(220A):6--25.

2. Broer, L. J. F. :Approximate equations for long water waves. Appl Sci Res,1975: (31): 337--396.

3. Kupershmidt, B. A.: Mathematics of dispersive waves. Comm Math Phys.1985: (99):51--73.

4. Wang, M.L.: A nonlinear function transform action and the exact solutions of the approximate equations for long waves in shallow water. Journal of Lan Zhou University. (Natural Sciences) 1998, 34 (2) : 21--25.

5. Huang, Z.H.: On Cauchy problems for the RLW equation in two space dimensions. Appl Math and Mech, 2002 : (23) 2:169--177.

6. Morlet ,J., Arens, G., Fourgeau, E.,Et al.: Wave propagation and sampling theory and complex waves.Geophysics, 1982, 47(2):222--236.

7. Wei, G. W.: Quasi wavelets and quasi interpolating wavelets. Chen Phys.Lett, 1998. 296(3 4): 215--222.

8. Wan, D. C. ,We,i G. W.: The Study of Quasi-Wavelets Based Numerical Method Applied to Burger' Equations. Appl.Math. Mech, 2000.(21) 1099. 